\title{
Cuestionario del rol docente en las organizaciones universitarias: diseño y validación en el marco del Espacio Europeo de Educación Superior
}

\author{
Mercedes Casañola Andrés, Claudio Carretero Chamarro, \\ Ángel Barrasa Notario, M. ${ }^{a}$ Victoria Sanagustin-Fons \\ casanola@unizar.es,ccar@unizar.es, abarrasa@unizar.es,vitico@unizar.es \\ Universidad de Zaragoza
}

Resumen. El propósito de esta investigación es diseñar y validar un cuestionario para medir las percepciones, actitudes y comportamientos del profesorado universitario respecto a su práctica docente y su relación con los estudiantes en el marco del Espacio Europeo de Educación Superior (EEES); considerando ciertos conceptos imprescindibles para el desempeño académico incardinados con el objetivo 4 de los Objetivos de Desarrollo Sostenible (ODS). El cuestionario se orienta a cumplir una doble finalidad, por un lado, posibilitar vías para incrementar la calidad de los procesos de enseñanzaaprendizaje mediante el autodiagnóstico de los docentes, $y$, por otro lado, orientar un camino de mejora continua e innovación metodológica y competencial. En un primer momento, se elaboró un borrador inicial de cuestionario con 11 dimensiones compuestas por 72 items, que, tras realizar un análisis psicométrico limitado, se redujo a únicamente 2 dimensiones con 20 items, que corresponde al cuestionario testado mediante una muestra de 360 docentes de universidades de toda España. La aplicación del análisis factorial sobre las respuestas arrojó tres factores con una alta fiabilidad: formación integral, formación en valores, innovación pedagógica y TIC. A la vista de los resultados, cabe inferir que este cuestionario posee una validez clara y fiable para ser utilizado en el ámbito universitario, dado que posee factores conectados estrechamente tanto con los principios del EEES como con el objetivo 4 de los ODS.

Palabras clave: rol docente; EEES; métodos de enseñanza; psicología educativa. 
Questionnaire ON THE ROLE OF TEACHING STAFF IN UNIVERSity ORGANIZATIONS: DESIGN AND VALIDATION IN THE FRAME OF THE EUROPEAN Higher Education Area

Abstract. The purpose of this research is to design and validate a questionnaire to measure the perceptions, attitudes and behaviors of university teaching staff regarding their teaching practice and their relationship with students in the framework of the European Higher Education Area (EHEA) and considering certain essential concepts for academic performance in line with goal 4 of the Sustainable Development Goals (SDGs). The questionnaire has two purposes: 1) to promote ways that teachers can engage in self-assessment and thus increase the quality of teaching-learning processes and 2) to help guide teachers on the path towards continuous improvement and methodological and competency innovation. The initial draft of the questionnaire had 11 dimensions consisting of 72 items, which after a limited psychometric analysis was reduced to only 2 dimensions with 20 items, corresponding to the questionnaire tested on a sample of 360 teachers from universities throughout Spain. Analysis of the responses revealed three factors with high reliability: comprehensive training; training in values; and pedagogical innovation and ICTs. The results indicate that this questionnaire has a clear and reliable validity for use in the university environment, given that it has factors closely connected both with the principles of the EHEA and with Goal 4 of the SDGs.

Keywords: teaching role; EHEA; teaching methods; educational psychology. 


\section{Planteamiento del problema}

Han transcurrido más de veinte años desde la Declaración de Bolonia (European Ministers in charge of Higher Education, 1999), punto de partida legal para el establecimiento y consolidación del Espacio Europeo de Educación Superior (Neave, 2003; Van der Wende, 2000; EURYDICE, 2020). Dicha Declaración de Bolonia consistió en una serie de principios destinados a armonizar los sistemas de educación superior de los países signatarios de la European Cultural Convention del Consejo de Europa, miembros de la Unión Europea (UE) y otros adheridos a dicho convenio (Beerkens, 2008; Alonso-Sáez y Arandia-Loroño, 2017). Entre los objetivos establecidos, destacan los siguientes: i) mejorar la movilidad geográfica de los graduados europeos, a través de un reconocimiento de sus titulaciones de forma ágil y recíproca por parte de los países que constituyen la UE; ii) establecer un sistema único de créditos, denominados ECTS (European Credit Transfer System), que incluyen el reconocimiento de la experiencia laboral y profesional de los estudiantes en la adquisición de competencias; iii) la transformación de los métodos de enseñanza-aprendizaje, que pasan a estar centrados en los estudiantes, los cuales se transforman en sujetos activos en su aplicación; iv) el cambio en el sistema de evaluación, convirtiéndose en continua, con las potencialidades de evaluación formativa asociada, y v) un enfoque de los estudios basados en la práctica, con la vista puesta en el perfil profesional que se plantea conseguir por parte de las diferentes instituciones de educación superior (Sanagustín-Fons, Cruz y Paricio, 2008; Sanagustín-Fons, Puyal, Moseñe y Tricas, 2007). Finalmente, destaca entre los objetivos el establecimiento de un marco común de cualificaciones para facilitar la comparabilidad entre los títulos obtenidos en diferentes universidades (Feeney y Hogan, 2017). Sin embargo, desde el punto de vista de la organización de la enseñanza en la educación superior, destaca el cambio de paradigma en el proceso de enseñanza-aprendizaje, donde el alumnado se convierte en el centro de este proceso. Además, se propugnó el cambio desde un entorno centrado en la transmisión de conocimiento a otro en el que las competencias se convierten en un valor primario de la evaluación de los resultados del aprendizaje (González et alii, 2003; Villarroel y Bruna, 2017). De tal forma, se estableció que los procesos de enseñanza-aprendizaje deberían orientarse a la adquisición de múltiples competencias, que previamente no se habían considerado como resultados que obtener en la enseñanza universitaria.

Finalmente, debe indicarse que, durante el establecimiento del EEES, comienzan a realizarse propuestas en relación con conseguir que los estudiantes alcancen, por un lado, las competencias específicas que tienen que ver con conocimientos tanto de tipo cognitivo, mecánico, técnico y de índole teórica como 
de índole práctica, es decir, se trata del saber hacer; además, se plantea que en el proceso de enseñanza-aprendizaje se alcancen, por otro lado, competencias transversales, con más contenido actitudinal, y que son altamente valoradas en el mercado laboral. Tales como la capacidad de trabajo en equipo, el denominado saber ser y el saber estar, que, principalmente, son las motivaciones laborales, la capacidad de adaptación y la resiliencia de los recursos humanos potenciales del mercado de trabajo (Sánchez, Amar y Triadú, 2018).

\section{Objeto de estudio}

Para adoptar un enfoque educativo alineado con los denominados principios de Bolonia y los ODS (ONU, 2015), es importante que el personal docente universitario adquiera las habilidades necesarias para llevar a cabo sus tareas docentes, lo que requiere una planificación organizacional adecuada que permita tanto la capacitación como el desarrollo personal de los profesores (Trigwell et alii, 2012; Wilkerson y Irby, 1998; Wilson y Berne, 1999; Zabalza y Beraza, 2003; Fernández-Ferrer y Miravalles, 2018). La evaluación del grado de adquisición de competencias profesionales por parte de los docentes debe llevarse a cabo tanto durante el proceso de aprendizaje como a través de los resultados de la práctica docente profesional que se puede medir en los resultados de aprendizaje de los estudiantes (Saroyan y Trigwell, 2015).

Existen pruebas del impacto que tiene una adecuada organización instruccional de los docentes en el desempeño del proceso de enseñanza-aprendizaje en las organizaciones de educación superior (Stes et alii, 2010). Además, la evaluación formativa de las competencias iniciales de estos posee una gran influencia en la realización posterior de las tareas docentes (Romero-Martín et alii, 2017). Es decir, el profesorado universitario debe adquirir toda una serie de herramientas docentes que resultan necesarias para la aplicación de los principios del proceso de convergencia europea, tanto desde el punto de vista de los métodos educativos, de enseñanza-aprendizaje, como desde la perspectiva de los inevitables y complejos procesos de evaluación. Actualmente, debido a la crisis provocada por la pandemia de la covid-19, la dificultad de establecer formas y criterios de evaluación justos $y$ adecuados va aumentando progresivamente. Asimismo, dicha tarea docente se torna más difícil, por la propia evolución de la sociedad del conocimiento y el uso de tecnologías de la comunicación y la información (Quiroz, y Quiroz, 2019; Falco, 2017; Pacheco, Ortega, Chong y Quiñonez, 2017; Granda, Espinoza y Espinoza, 2019; García-Cabrero, Luna-Serrano, Ponce-Ceballos, Cisneros-Cohenour, Cordero-Arroyo, Espinoza-Díaz y García-Vigil, 2018). Es precisamente en este entorno en el que se esboza el objeto de estudio de nuestra 
investigación, planteando que se pueda llevar a cabo una mejor autoevaluación por parte del profesorado de las instituciones de educación superior, para que se enfoquen en reconocer sus puntos débiles y determinar sus puntos fuertes, con el propósito de establecer programas de formación continua más eficientes, así como enfocar los sistemas de gestión de la calidad total que actualmente se aplican en las universidades.

\section{Fundamentación teórica}

La perspectiva teórica desde la que se aborda esta investigación parte de la sociología de las organizaciones (Lucas Marín y García Ruiz, 2005; Brunet y Vidal, 2004, Sanagustín-Fons y Brunet, 2017), en el sentido de entender la actividad docente incardinada en el ámbito de las organizaciones universitarias, siendo los actores sociales principalmente implicados, los docentes y los discentes, además del resto de agentes socioeducativos y sociopolíticos presentes en el funcionamiento del sistema educativo universitario. Siendo la principal finalidad de dichas organizaciones proponer elementos facilitadores en la gestión del conocimiento, considerando al docente como un agente de cambio social; orientando la organización educativa a estrechar relaciones con el sector social y productivo para promover el bien común y el desarrollo social (Bonilla, Badillo y Genis, 2019), así como una educación de calidad para todos, como fija el objetivo número 4 de los ODS de la Agenda 2030 de Naciones Unidas (ONU, 2015). Observándose, además, toda una serie de conceptos teóricos que influyen claramente en los procesos internos de dichas organizaciones y en su codeterminación con el entorno social, económico, tecnológico e ideológico-cultural (Perrow, 1990). De ahí que, con el objetivo de cambiar la forma en la que se aborda el paradigma establecido y la propia cultura organizacional (Hofstede, 1998; Schein, 2010) en el considerado como Espacio Europeo de Educación Superior y bajo el paraguas del objetivo 4 de los ODS (ONU, 2015), es necesario señalar los principales valores que emergen de dicho cambio. Se podrían considerar como principales valores los que están relacionados con una educación orientada a la práctica; que responde de forma adecuada y eficiente a las demandas del entorno; con un enfoque centrado en los estudiantes y que rinde cuentas a la sociedad por todo lo que se lleva a cabo en el interior de la organización educativa, orientando sus esfuerzos a alcanzar cotas de calidad y excelencia en los procesos de gestión del conocimiento y su aplicación en la sociedad (Peralta, Stefanel, Cervantes y Salgado, 2018; Esquivel, León y Castellanos, 2017).

Asimismo, es imprescindible profundizar en las motivaciones del profesorado universitario, dado que se trata de uno de sus principales protagonistas y agentes 
de cambio social. Cabe señalar, además, que el enfoque del profesor depende en gran medida de su actitud hacia la temática y el entorno en el que enseña (Prosser et alii, 2005; Imbermón, 2017), los académicos que experimentan sus materias de acuerdo con una menor estructura integradora experimentan su enseñanza de una manera más centrada exclusivamente en el profesor, mientras que aquellos con una experiencia más holística se centran en la comprensión global de la materia que abordar (Salgueiro, Sabelli, Manzi, Irigoyen, Exeni, Vidal y Bono, 2021).

Como desempeño laboral, la docencia es una tarea compleja, precisamente por su multidimensionalidad, pues incluye, entre otras, el conocer profundamente las materias específicas de cada ámbito profesional; saber transmitir dichos conocimientos; tener habilidades comunicativas específicas; poseer competencias de evaluación de los conocimientos adquiridos por parte del estudiantado; además de ofrecer una educación integral y en valores, entre otras dimensiones (Sanagustín-Fons, 2008). Además, se encuentra sometida a cambios constantes, dadas las especificidades tanto de los discentes como de la estructura de la organización universitaria, la evolución social y el desarrollo y aplicación de las tecnologías que tanto afectan a su práctica diaria (Walker, 2015; 2020). Además de ser una actividad marcada por el grado de implicación y vocación del profesorado, Lucarelli (2004) hace referencia a la importancia que posee la construcción de la identidad de dicho profesorado en el proceso de su desempeño (Alcalá del Olmo, 2019). Es así cómo se entiende la importancia que posee dicha actividad para el conjunto del desarrollo de los futuros profesionales y de los avances en el conocimiento. Por otro lado, tal y como señala Herzberg (2017), cuando la tarea entusiasma en sí misma, es cuando los trabajadores son capaces de involucrarse al máximo, promoviendo, en este caso, el desarrollo de los procesos de enseñanzaaprendizaje de la mejor forma posible. Entonces surgen las dos principales cuestiones, ¿es la práctica de enseñar? o ¿es la práctica específica para la cual fue formado el profesorado en su campo particular? Siendo ambas cuestiones una de las claves esenciales para comprender la esencia de la docencia universitaria. Resulta también importante considerar las voces críticas que establecen algunos de los problemas a los que se enfrenta, en la actualidad, la práctica de la docencia universitaria; se trata de una sustracción progresiva de cualidades que ha conducido a los docentes a la pérdida de control y sentido sobre el propio trabajo (Contreras, 2018; García, 2020). Trigwell (2012) indica que hay relaciones significativas entre las formas en que los profesores experimentan emocionalmente el contexto de la enseñanza y, por otro lado, las formas en las que abordan su enseñanza junto con las emociones positivas que se asocian al alumno como protagonista del proceso de enseñanza-aprendizaje. Otros autores subrayan, precisamente, la im- 
portancia que posee la gestión adecuada de las emociones en la labor del docente universitario, principalmente, para ser capaz de relacionarse de forma auténtica con sus estudiantes, eliminando sesgos de edad, cosmovisiones y otros valores culturales (Tacca Huamán, Tacca Huamán y Cuarez Cordero, 2020; Llorent, Zych y Varo-Millán, 2020).

En numerosas ocasiones, la evaluación de las tareas docentes se lleva a cabo por los estudiantes como agentes finalistas en el sistema universitario (Ion et alii, 2016; Van der Lans et alii, 2015). En otros casos, se proponen una serie de herramientas para que los docentes aprendan tanto las motivaciones propias (Moskal et alii, 2016; Visser-Wijnveen et alii, 2011) como las habilidades necesarias para realizar sus tareas docentes de forma satisfactoria (Díaz et alii, 2016; Koloi-Keaikitse, 2017; Muñoz et alii, 2011; Tigelaar et alii, 2004; Van den Bos y Brouwer, 2014). Además, por la importancia que están adquiriendo recientemente, deben destacarse el impacto y la percepción que los docentes poseen respecto al uso y aplicación de TIC en su desempeño (Bas et alii, 2016; Ertmer et alii, 2012; Fernández-Batanero y Ruiz, 2015; Hernández-Ramos et alii, 2012; Scherer et alii, 2015; Tejedor et alii, 2009; Fernández y Pérez, 2018), dado que pueden implicar un claro aumento en la productividad docente debido a la simplicidad de la enseñanza (Englund et alii, 2017; Mishra y Koehler, 2006; Muñoz-Repiso y Tejedor, 2012). Características necesarias para una formación integral del profesorado, la adquisición de los valores acordes al EEES y a los ODS y una adaptación a la innovación tecnológica y todo lo que supone en los procesos de enseñanzaaprendizaje.

\section{Diseño y metodología}

\subsection{Diseño del cuestionario}

Desde la necesidad de impulsar toda la cultura de educación orientada a la excelencia, para una mejora continua de la enseñanza, esta investigación se centra en la creación y validación de un cuestionario que ha sido denominado Cuestionario del Rol Docente en el Espacio Europeo de Educación Superior (CRD-EEES). Este cuestionario responde a una demanda real que aumenta cada vez más en la universidad. Esta herramienta pretende valorar el entusiasmo y el deseo del docente por contribuir a una educación universitaria de calidad, consolidar un paradigma común que ofrezca flexibilidad a los estudiantes, así como respuestas a las actuales demandas sociales en un entorno abierto y dinámico.

El objetivo principal de este cuestionario consiste en la autoevaluación del profesor universitario, detectando fortalezas, áreas de mejora y potencialidades, 
en el sentido de plantear una mejora continua en el desempeño docente y su impacto en la formación de los estudiantes. El objetivo principal de este cuestionario consiste en la autoevaluación del profesor universitario, detectando fortalezas, áreas de mejora y potencialidades en el sentido de plantear una mejora continua en el desempeño docente y su impacto en la formación de los estudiantes, pero también en su rol investigador, así como una cierta ambigüedad de rol que se manifiesta en muchas ocasiones, específicamente, a lo largo del año 2020, en el que los docentes se han visto sometidos a nuevas exigencias consecuencia de la crisis generada por la pandemia de la covid-19 (Surdez, Magaña y Sandoval, 2017; Lemos, Calle, Roldán, Valencia, Orejuela y Román-Calderón, 2019; Oros, Vargas y Chemisquy, 2020). Teniendo en cuenta la idiosincrasia y la propia historia de los diferentes contextos asociados al sistema de enseñanza tradicional, todavía presente en ciertos ámbitos y universidades españolas.

La creación del CRD-EEES, se inicia con el consenso, asesoramiento y orientación de profesionales de la pedagogía y la psicología educacional, junto con una cantidad considerable de documentos de fuentes básicas y fiables, en consonancia con los enfoques metodológicos sugeridos en las declaraciones del Plan de Bolonia y el Proyecto Tuning (Tuning Educational Structures in Europe, 2006); asimismo, se ha considerado el seguimiento y evaluación del EEES que aparece en el informe titulado The European Higher Education Area in 2020: Bologna Process Implementation Report (EURYDICE, 2020).

Se inicia así el arranque de la elaboración de una batería de cuestiones que son reagrupadas, considerando lo que pretenden medir, según diferentes dimensiones. Este primer listado de cuestiones fue propuesto a un panel de diez expertos, siguiendo el método Delphi, que eran profesores de universidad de distintas áreas de conocimiento. Los miembros del panel realizaron un análisis en profundidad, aglutinando las cuestiones o ítems en distintas dimensiones según su coherencia, significación e impacto. La determinación de las dimensiones de mayor importancia que se incluyen en el cuestionario definitivo parte de varias premisas: i) el marco competencial del docente en el EEES; ii) el estudiante como protagonista de la mejora de la calidad evaluadora; iii) las metodologías aplicadas por el docente $y$, finalmente, iv) la idiosincrasia de este en su particular modo de ejercer la docencia (Aparicio Arias, 2011; Walker, 2020).

Tras el esfuerzo y tiempo invertidos en la reflexión, discusión, análisis y maduración, por parte del panel de expertos, y de la discusión del grupo de investigación de las dimensiones e ítems que potencialmente conformarían el CRDEEES, se definió un primer cuestionario preliminar cuya estructura responde a la establecida en la tabla 1 , donde se indican las 11 dimensiones en que se inscribían un total de 72 elementos. 
Tabla 1. Dimensiones de la versión preliminar del cuestionario

\begin{tabular}{|c|l|}
\hline Número & Etiqueta \\
\hline 1 & Actitud hacia el EEES \\
2 & Actitud de colaboración \\
3 & Papel del guía y mentor \\
4 & Aprendizaje autodirigido \\
5 & Competencias en tecnologías de la información \\
6 & Personalización de la enseñanza \\
7 & Educación integral \\
8 & Flexibilidad \\
9 & Evaluación \\
10 & Desarrollo personal del docente \\
11 & Innovación y transformación \\
\hline
\end{tabular}

Fuente: elaboración propia.

La siguiente fase, totalmente cuantitativa, se centró en la aplicación y el análisis de la validez y fiabilidad del CRD-EEES desde un punto de vista psicométrico. Con este propósito, una muestra de 42 profesores universitarios respondieron a cuestiones referidas a la medida que proporcionaba cada ítem, su relación con una de las dimensiones y la significatividad de dichas dimensiones dentro del marco del EEES y los ODS. La escala definida de 11 dimensiones no tenía consistencia interna, pues se quedó por debajo de los límites para su validación estadística, que, tras aplicar el correspondiente análisis factorial utilizando rotación Varimax, mostró que dos de los factores explicaban el 22,50\% y el 21,97\% de la varianza total, respectivamente. Estos factores fueron denominados: formación integral, que estaba compuesto por 13 preguntas, e innovación pedagógica y uso de las TIC, que constaba de 7 elementos. La versión preliminar del cuestionario, que consta de 20 elementos repartidos en dos factores, constituyó la primera definición del CRD-EEES.

En el presente estudio queremos validar la herramienta con una muestra mucho más representativa, con objeto de lograr una mayor validez y confiabilidad, así como consolidar el CRD-EEES como un instrumento creado, validado y disponible para la comunidad educativa y conseguir una medida de la calidad en el proceso de enseñanza-aprendizaje.

Se realizó la aplicación de este cuestionario a una muestra de 360 profesores de diferentes grados, categorías y centros académicos de varias comunidades autónomas (Aragón, Cataluña, Galicia y el País Vasco, principalmente), con 
pruebas psicométricas más completas. La aplicación del cuestionario en dicha muestra ha arrojado resultados significativos y contrastables, que permiten destacar la aparición de un nuevo factor: la formación transversal, agregada a los ya existentes, formación integral e innovación pedagógica y TIC, lo que enriquece la estructura del cuestionario, así como la eliminación de 4 elementos que no estaban proporcionando información fiable. El cuestionario se construyó finalmente con 16 ítems con una elevada concreción (anexo I).

A través de esta investigación, se ha validado el CRD-EEES. En el nivel metodológico, el objetivo de su aplicación en las aulas a través de la figura guía, facilitadora e impulsora del docente, se considera indispensable para alcanzar así un óptimo nivel de calidad y excelencia universitarias. Además, el presente trabajo supone de una manera sencilla, rápida y ágil la mejora continua de los procesos educativos en el interior de las organizaciones de educación superior, las cuales se hayan inmersas en evaluaciones de su calidad, excelencia y eficiencia; además, de las exigencias de rendición de cuentas que la propia normativa europea e internacional exige (Rodríguez-Gómez, 2019; Sisto, 2020; Sanagustín-Fons y Brunet, 2017).

\section{Versión reducida del cuestionario}

El CRD-EEES, se administró junto con otro cuestionario: el Multifactor Leadership Questionnaire, en su formato 5X (Avolio et alii, 2004), adaptado al contexto universitario.

Al final del cuestionario de la función docente, se solicitan datos de interés para un análisis posterior: sexo, edad, universidad de pertenencia, departamento, asignatura impartida y categoría laboral. El cuestionario está diseñado para facilitar al máximo sus respuestas. Destacando el anonimato y la confidencialidad de la encuesta, así como la importancia de la sinceridad en la respuesta para optimizar la validez de la información. Los elementos dispuestos por los dos factores que se extrajeron del primer análisis, 13 ítems para el factor de formación integral y 7 ítems para innovación pedagógica y TIC, según se muestra en la tabla 2. Los resultados de las repuestas proporcionadas por 360 profesores universitarios fueron analizados usando SPSS Versión 20 para Windows. 


\section{Tabla 2. Versión preliminar del cuestionario}

\begin{tabular}{|c|c|}
\hline Ítem & Formación integral \\
\hline 1 & $\begin{array}{l}\text { Intento desarrollar diversos roles de interacción y fomento de la participación de los } \\
\text { estudiantes. }\end{array}$ \\
\hline 2 & $\begin{array}{l}\text { Manejo diferentes estrategias metodológicas que mejoran la participación y el trabajo } \\
\text { autónomo de los estudiantes. }\end{array}$ \\
\hline 3 & $\begin{array}{l}\text { Incluyo en mi enseñanza de forma indirecta valores típicos basados en los valores } \\
\text { democráticos. }\end{array}$ \\
\hline 4 & $\begin{array}{l}\text { Evalúo en igual intensidad tanto el aprendizaje adquirido por el alumno y los procesos } \\
\text { para adquirirlos. }\end{array}$ \\
\hline 5 & $\begin{array}{l}\text { Creo que estoy preparado para personalizar el proceso de enseñanza-aprendizaje depen- } \\
\text { diendo de la diversidad inicial de los estudiantes. }\end{array}$ \\
\hline 6 & $\begin{array}{l}\text { Me considero flexible y abierto a la hora de marcar el ritmo de actividades de enseñanza, } \\
\text { incluidas formas colaborativas e interactivas. }\end{array}$ \\
\hline 7 & $\begin{array}{l}\text { Mis sistemas de evaluación recogen resultados tanto de la asimilación de conceptos y } \\
\text { como de la persistencia del desarrollo de habilidades y actitudes. }\end{array}$ \\
\hline 8 & $\begin{array}{l}\text { Organizo el espacio de enseñanza (o contexto de aprendizaje) de una investigación enfo- } \\
\text { cada en que los estudiantes tienen el desafío de descubrir o responder a algunas hipótesis } \\
\text { o preguntas que planteo. }\end{array}$ \\
\hline 9 & $\begin{array}{l}\text { Promuevo en los estudiantes su autocrítica y autoevaluación en las actividades que real- } \\
\text { izan con mi sistema educativo. }\end{array}$ \\
\hline 10 & $\begin{array}{l}\text { Mis objetivos docentes promueven el desarrollo integral y mejorar el compromiso social } \\
\text { ético de los futuros profesionales. }\end{array}$ \\
\hline 11 & Inculco valores universales en la educación universitaria de mis alumnos. \\
\hline 12 & $\begin{array}{l}\text { Aplico las líneas pedagógicas y las técnicas metodológicas que enseñan cómo llevar a } \\
\text { cabo una educación integral. }\end{array}$ \\
\hline 13 & Combino diferentes estilos de enseñanza para reforzar varias formas de aprendizaje. \\
\hline Ítem & Innovación pedagógica TIC \\
\hline 14 & $\begin{array}{l}\text { Me preocupa actualizar mi conocimiento sobre las TIC (tecnologías de la información } \\
\text { y la comunicación). }\end{array}$ \\
\hline 15 & $\begin{array}{l}\text { Intento acercar mis intereses de investigación a los estudiantes con rigor y veracidad de } \\
\text { los problemas y enfoques actuales. }\end{array}$ \\
\hline 16 & Aplico TIC que facilitan el aprendizaje. \\
\hline 17 & Considero que mi estilo de enseñanza es reflexivo y transformador. \\
\hline 18 & $\begin{array}{l}\text { Conozco la forma obtener y desarrollar la tecnología para la formación integral de los } \\
\text { estudiantes. }\end{array}$ \\
\hline 19 & $\begin{array}{l}\text { Tengo referencias destacables a buenas prácticas como resultado de mi estilo de en- } \\
\text { señanza innovador. }\end{array}$ \\
\hline 20 & Integro el derecho a la educación a lo largo de la vida con diversos recursos de e-learning. \\
\hline
\end{tabular}

Fuente: elaboración propia. 


\subsection{Metodología}

\section{Muestra}

La muestra de participantes estuvo compuesta por 360 profesores universitarios de 109 departamentos, por lo tanto, las muestras cubren profesorado de un amplio rango de áreas. Esta muestra fue reclutada al azar a través de correo masivo, con edades comprendidas entre 23 y 78 años (mediana $=44,73$ años, desviación estándar $=10,13$ años), siendo 205 hombres $(56,94 \%)$ y 155 mujeres $(43,06 \%)$. La muestra se clasificó en tres categorías: personal funcionario, profesor contratado a tiempo completo y personal contratado a tiempo parcial, con la distribución que se muestra en la tabla 3.

Tabla 3. Composición de la muestra

\begin{tabular}{|l|c|c|c|c|}
\hline & $\begin{array}{c}\text { Cuerpos } \\
\text { docentes }\end{array}$ & $\begin{array}{c}\text { Contratados } \\
\text { a tiempo completo }\end{array}$ & $\begin{array}{c}\text { Contratados } \\
\text { a tiempo parcial }\end{array}$ & Total \\
\hline Mujer & 60 & 57 & 38 & 155 \\
\hline Hombre & 71 & 94 & 40 & 205 \\
\hline Total & 131 & 151 & 78 & 360 \\
\hline
\end{tabular}

Fuente: elaboración propia.

La aplicación del análisis factorial exploratorio se ha realizado según la secuencia de pasos que se especifica a continuación. En primer lugar, se ha realizado el cálculo de la matriz de correlación entre las variables de datos originales, a la que se han aplicado un conjunto de pruebas estadísticas para verificar que sea significativamente diferente de una matriz de identidad. En caso de la confirmación de una identidad matriz de correlación, los elementos no estarían correlacionados, por lo que el conjunto bajo estudio no puede ser factorizado. Seguidamente, se ha procedido a obtener los factores iniciales para, finalmente, proceder a su rotación con objeto de representar los datos originales de una forma más eficiente. Después de analizar la matriz de correlación y obtener los factores, se han analizado las comunalidades, la varianza total explicada por cada factor y el gráfico de sedimentación, con objeto de extraer aquellos factores que tienen una capacidad explicativa significativa.

Para verificar que los datos se ajustan a una descomposición factorial, se han aplicado las siguientes pruebas estadísticas para asegurar que la matriz de correlaciones no está mal condicionada: el determinante de la matriz, la prueba KMO y la prueba de esfericidad de Bartlett. 
El determinante de la matriz de correlación es menor que 0,050 cuando las correlaciones cruzadas de algunos elementos son altos, pero no puede ser igual a 0 , puesto que en este caso la matriz de correlaciones no sería invertible, por lo que no podría realizarse la descomposición en factores. En nuestro caso, el determinante de la matriz de correlación es menor que 0,001 , pero distinto de 0 , en consecuencia, la descomposición factorial puede aplicarse y existen indicios de que un número bajo de estos tiene un elevado poder explicativo.

La prueba de Bartlett se ha aplicado para verificar la homocedasticidad de la matriz de correlaciones, es decir, que la varianza asociada a esta es homogénea. El estadístico de Bartlett obedece a una distribución chi cuadrado. Los resultados se podrían considerar adecuados para el análisis si se rechaza la hipótesis nula de homocedasticidad con una significatividad debajo de 0,05 , que en los datos analizados se observa con claridad al rechazarse dicha hipótesis con una significatividad inferior a 0,001 , por lo que unos elementos presentarán un poder explicativo superior a los restantes.

La prueba de Kaiser-Meyer-Olkin (KMO) se usa para comparar los coeficientes de correlación y los valores de correlaciones parciales. Esta prueba estadística tiene un rango de variación comprendido entre 0 y 1 . Los contrastes estadísticos cercanos a 0 implican una baja correlación entre parejas de variables, cuya consecuencia es la inviabilidad de realizar una descomposición en factores. Por el contrario, los valores de las estadísticas de prueba cercanas a 1 indican unas buenas condiciones para la aplicación del análisis factorial. En el caso bajo análisis, el contraste estadístico KMO obtenido es 0,911 , por lo que resulta adecuado aplicar una descomposición factorial a los datos.

Los factores principales se extraen del conjunto de variables mediante el análisis por componentes principales (PCA), donde los componentes se ordenan dependiendo de la varianza explicada aplicando la diagonalización de la matriz de covarianzas, siendo la primera componente una combinación de elementos con poder explicativo de la mayor parte de la varianza, la segunda componente tiene el poder explicativo de la mayor parte de la varianza restante, y así sucesivamente. Para determinar el número de factores óptimos, se utilizó el criterio clásico de selección de componentes con cargas superiores a 1 (Kaiser, 1960; 1970). En este caso, se obtuvieron tres factores con cargas 7,87, 1,83 y 1,33, respectivamente, correspondiendo el porcentaje explicado de varianza al $60 \%$ del total, que resulta más que aceptable dado el elevado número de factores iniciales.

Seguidamente, se ha procedido a evaluar las comunalidades de los elementos de cada uno de los tres factores extraídos anteriormente. A partir de los resultados obtenidos, se ha procedido a eliminar cuatro de los elementos de la versión 
inicial del cuestionario, correspondientes al ítem 7, ítem 13, ítem 15 e ítem 19, debido a que los valores de sus comunalidades, que correspondían a 0,343,0,411, 0,406 y 0,335 , respectivamente, tenían un valor más reducido que las restantes, que, como sucede en este caso, eran inferiores al umbral de 0,5 que marca el límite para ser considerados significativos. Por tanto, después de aplicar la eliminación de los elementos menos significativos, el cuestionario estaría compuesto de 16 elementos repartidos entre 3 factores explicativos.

Finalmente, los factores se rotan aplicando el método Oblimin con objeto de relacionar de forma más cercana los factores con la información proporcionada por cada uno de los elementos del cuestionario. Los elementos del cuestionario se distribuyen según tres factores, en la forma mostrada en la tabla 4, donde se puede observar que el primer factor incluye 8 elementos y los otros dos se componen de 4 elementos cada uno. El tercer factor extraído en el análisis factorial actualiza el sentido de la dimensión antes mencionada, cuyo nombre se mantiene. El primer y el segundo factores extraídos tras rotar la matriz de correlación se derivan, principalmente, de las anteriores a las primeras dimensiones, y se denominan: formación integral, que está formado por los elementos iniciales $1,2,4,5$, $6,8,9$ y 17 , y formación transversal, que corresponde con los elementos iniciales $3,10,11,12$. Por último, el tercer factor consiste en los elementos iniciales 14, 16, 18 y 20 , alineados con los siete elementos iniciales que componían la dimensión innovación pedagógica y TIC de la versión preliminar del cuestionario, de donde se han eliminado los ítems 13 y 19, y el ítem 17 pasó a pertenecer al primer factor. La tabla 4 muestra que las cargas de las variables son mayores que 0,600 en cualquiera de los ítems para el factor al que se asignan, concluyendo que la descomposición en los factores obtenida es adecuada. 
Tabla 4. Rotación de la matriz de componentes

\begin{tabular}{|c|c|c|c|}
\hline \multicolumn{3}{|c|}{ Factor } \\
\hline Ítem & Formación integral & Formación transversal & $\begin{array}{c}\text { Innovación pedagógica } \\
\text { TIC }\end{array}$ \\
\hline 2 & 0,705 & 0,041 & 0,255 \\
\hline 1 & 0,684 & 0,235 & 0,162 \\
\hline 9 & 0,679 & 0,187 & 0,047 \\
\hline 8 & 0,668 & 0,292 & 0,077 \\
\hline 17 & 0,624 & 0,171 & 0,357 \\
\hline 5 & 0,615 & 0,205 & 0,211 \\
\hline 6 & 0,612 & 0,148 & 0,247 \\
\hline 4 & 0,594 & 0,375 & 0,170 \\
\hline 11 & 0,156 & 0,817 & 0,220 \\
\hline 10 & 0,332 & 0,804 & 0,063 \\
\hline 3 & 0,205 & 0,799 & 0,091 \\
\hline 12 & 0,405 & 0,621 & 0,293 \\
\hline 14 & 0,068 & 0,058 & 0,876 \\
\hline 16 & 0,200 & 0,073 & 0,843 \\
\hline 18 & 0,350 & 0,168 & 0,692 \\
\hline 20 & 0,156 & 0,296 & 0,631 \\
\hline
\end{tabular}

Fuente: elaboración propia.

Después de aplicar el análisis factorial, se han obtenido las varianzas explicadas totales de los tres factores resultantes de la extracción con rotación Oblimin. La extracción de la varianza total explicada por cada uno de los tres factores de la matriz no girada es $6,52,1,80$ y 1,31 , respectivamente, siendo las varianzas extraídas de las restantes componentes menores que 1 . La rotación de la matriz de correlación proporciona la misma varianza explicada, pero la distribución entre los factores se vuelve más uniforme, como se puede ver en el hecho de que cada uno de ellos contribuyó al 40,78\%, 11,23\% y 8,21\%, respectivamente, para los factores iniciales, y se convirtió en $24,36 \%, 17,96 \%$ y $17,80 \%$, respectivamente, para cada uno de los tres factores obtenidos de la matriz rotada, cuyos valores de la varianza explicada son $3,99,2,87$ y 2,86 , respectivamente, por lo que se observa un elevado grado de uniformización de estos.

La consistencia interna del cuestionario se verifica mediante la prueba alfa de Cronbach. Se han alcanzado grandes valores cercanos a 1 para los tres factores del cuestionario: el alfa de Cronbach del factor formación integral, compuesto 
por 8 ítems, es 0,852; el alfa de Cronbach del factor innovación pedagógica y TIC, compuesto por 4 ítems, es 0,815, y el alfa de Cronbach del factor formación transversal, compuesto de 4 ítems, es 0,851 , por lo que la fiabilidad del cuestionario parece validada.

Finalmente, para determinar la interrelación entre los problemas de los diferentes factores se han calculado los valores de las relaciones cruzadas entre los diferentes conglomerados de cuestiones. Las correlaciones observadas son positivas en todos los casos, por lo que la escala de Likert para los conglomerados de cuestiones parece haberse definido en el sentido más apropiado. Se observa que la correlación cruzada entre los factores innovación pedagógica y TIC y los otros dos factores, formación integral y formación transversal, tiene correlaciones cruzadas de bajo valor, iguales a 0,366 y 0,256, respectivamente. Por otro lado, la correlación cruzada entre los factores formación integral y formación transversal tiene un valor ligeramente más alto que los anteriores, al ser igual a 0,500 , pero permanece en valores moderados.

\section{Discusión y conclusiones}

Desde la perspectiva teórica de la sociología de las organizaciones de educación superior, se propone un instrumento de medida diseñado para que su aplicación permita llevar a cabo un autodiagnóstico del profesorado; consiguiendo un mayor conocimiento de este en su tarea universitaria, de tal forma que se establezcan estrategias que permitan adquirir una serie de valores que respondan a las exigencias de la sociedad. La complejidad de nuestra sociedad del siglo xxi implica que la gestión del conocimiento y la labor del profesorado universitario están sometidas a nuevas presiones procedentes de la evolución tecnológica y social, y, al igual que para otros autores, eso se refleja en nuestra propuesta (García Peñalvo y Rodríguez Conde, 2020; Peralvo y Merino, 2018). En esta investigación se ha dejado patente que la necesaria implicación del profesorado en su desempeño laboral es imprescindible para que se puedan alcanzar los valores del EEES y del objetivo 4 de los ODS, tal y como otros autores lo han identificado (Vergara y Núñez, 2021; Martínez, Llorens, Rodríguez-Lifante, Iniesta, Ramos y Ferreira, 2020). Igualmente, se deben generar espacios de formación profesional que permitan el desarrollo tanto técnico-académico como personal y socioemocional de los docentes, algo que se descubre en nuestra investigación y se corrobora en otras indagaciones relacionadas (Regueira y Gewerc, 2020; Llorent et alii, 2020; Jiménez-Hernández, González-Ortiz y Tornel-Abellán, 2018). Así, ya no solamente es necesario tener vocación sino que esta debe ir acompañada por toda una serie de competencias adquiridas que doten a la labor docente de todo su sentido. 
Mejorar los procesos de enseñanza-aprendizaje implica alcanzar una educación de calidad, inclusiva, e indirectamente lograr mayor desarrollo social y una mejor sociedad; coincidimos así con estudios previos como los de Hernández-Sánchez y Sánchez-García (2018), Alcaín Martínez y Medina-García (2017) y LunaNemecio (2019).

Este estudio permite concluir que el cuestionario CDR-EEES es una herramienta psicométrica adecuada para medir las competencias en formación integral, formación transversal, e innovación pedagógica y TIC en los docentes, herramientas imprescindibles para que los valores de la convergencia europea $y$ del objetivo 4 de los ODS formen parte del quehacer universitario. Por lo tanto, este instrumento se presenta como una referencia de uso en este ámbito. Además, se concluye que su utilidad es elevada en tres ámbitos concretos: en primer lugar, en el ámbito psicológico, enfoque micro y más psicológico, permitiendo el autoconocimiento de los docentes universitarios, siendo una herramienta que puede ayudar a la mejora de su autoestima y, por tanto, de su desempeño y aceptación social, por parte del estudiantado; en segundo lugar, en la gestión de la organización, enfoque meso, más psicosociológico, para su uso en los sistemas de gestión de la calidad total, que plantean la mejora continua de los procesos de enseñanza-aprendizaje en las universidades españolas, y, a través de los cuales, se está exigiendo una rendición de cuentas y una eficiencia en su funcionamiento, y, finalmente, en la investigación, enfoque macro que permita estudiar y analizar el grado de interiorización de los principios del EEES por parte de los docentes de las organizaciones universitarias en España, permitiendo un autodiagnóstico que ayude a buscar causas y consecuencias de la situación analizada. Se concluye, finalmente, que las áreas de mejora del personal de las instituciones universitarias necesitan que su personal adquiera competencias y habilidades que favorezcan su desempeño académico, su bienestar y calidad de vida laboral, con el objetivo de alcanzar un círculo virtuoso docente-discente. 


\section{Anexo I. Cuestionario del Rol Docente en el Espacio Europeo de Educación Superior (CRD-EEES)}

Indique su respuesta para cada ítem.

$1=$ nunca $; 2$ raramente 3 = ocasionalmente $4=$ frecuentemente; 5 = casi siempre.

Su participación es sinceramente apreciada. Muchas gracias

\begin{tabular}{|c|c|c|c|c|c|c|}
\hline Ítem & Cuestión & & & & & \\
\hline q1 & $\begin{array}{l}\text { Incluyo en mi enseñanza de forma indirecta, valores típicos basa- } \\
\text { dos en los valores democráticos. }\end{array}$ & 1 & 2 & 3 & 4 & 5 \\
\hline q2 & $\begin{array}{l}\text { Intento desarrollar diversos roles de interacción y fomento de la } \\
\text { participación de los estudiantes. }\end{array}$ & 1 & 2 & 3 & 4 & 5 \\
\hline q3 & $\begin{array}{l}\text { Me preocupa actualizar mi conocimiento sobre las TIC (tec- } \\
\text { nologías de la información y la comunicación). }\end{array}$ & 1 & 2 & 3 & 4 & 5 \\
\hline $\mathrm{q} 4$ & $\begin{array}{l}\text { Manejo diferentes estrategias metodológicas que mejoran la par- } \\
\text { ticipación y el trabajo autónomo de los estudiantes. }\end{array}$ & 1 & 2 & 3 & 4 & 5 \\
\hline q5 & $\begin{array}{l}\text { Mis objetivos docentes promueven el desarrollo integral y mejo- } \\
\text { ran el compromiso social y ético de los futuros profesionales. }\end{array}$ & 1 & 2 & 3 & 4 & 5 \\
\hline q6 & $\begin{array}{l}\text { Evalúo en igual intensidad tanto el aprendizaje adquirido por el } \\
\text { alumno como los procesos para adquirirlos. }\end{array}$ & 1 & 2 & 3 & 4 & 5 \\
\hline q7 & Aplico TIC que facilitan el aprendizaje. & 1 & 2 & 3 & 4 & 5 \\
\hline q8 & $\begin{array}{l}\text { Creo que estoy preparado para personalizar el proceso de en- } \\
\text { señanza-aprendizaje dependiendo de la diversidad inicial de los } \\
\text { estudiantes. }\end{array}$ & 1 & 2 & 3 & 4 & 5 \\
\hline q9 & $\begin{array}{l}\text { Inculco valores universales en la educación universitaria de mis } \\
\text { estudiantes. }\end{array}$ & 1 & 2 & 3 & 4 & 5 \\
\hline q10 & $\begin{array}{l}\text { Me considero flexible y abierto a la hora de marcar el ritmo de } \\
\text { actividades de enseñanza, incluidas formas colaborativas e inter- } \\
\text { activas. }\end{array}$ & 1 & 2 & 3 & 4 & 5 \\
\hline q11 & $\begin{array}{l}\text { Sé cómo obtener y desarrollar la tecnología para la formación in- } \\
\text { tegral de los estudiantes. }\end{array}$ & 1 & 2 & 3 & 4 & 5 \\
\hline $\mathrm{q} 12$ & $\begin{array}{l}\text { Organizo el espacio de enseñanza (o contexto de aprendizaje) } \\
\text { con un enfoque en el que los estudiantes tienen el desafío de des- } \\
\text { cubrir o responder a algunas hipótesis o preguntas que planteo. }\end{array}$ & 1 & 2 & 3 & 4 & 5 \\
\hline q13 & $\begin{array}{l}\text { Aplico las líneas pedagógicas y las técnicas metodológicas que en- } \\
\text { señan cómo llevar a cabo una educación integral. }\end{array}$ & 1 & 2 & 3 & 4 & 5 \\
\hline
\end{tabular}




\begin{tabular}{|l|l|l|l|l|l|l|}
\hline q14 & $\begin{array}{l}\text { Promuevo en los estudiantes su autocrítica y autoevaluación en } \\
\text { las actividades que realizan con mi sistema educativo. }\end{array}$ & 1 & 2 & 3 & 4 & 5 \\
\hline q15 & $\begin{array}{l}\text { Integro el derecho a la educación a lo largo de la vida con diversos } \\
\text { recursos de e-learning. }\end{array}$ & 1 & 2 & 3 & 4 & 5 \\
\hline q16 & $\begin{array}{l}\text { Considero que mi estilo de enseñanza es reflexivo y transforma- } \\
\text { dor. }\end{array}$ & 1 & 2 & 3 & 4 & 5 \\
\hline
\end{tabular}

Fuente: elaboración propia.

\section{Referencias}

Alcaín Martínez, E. y Medina-García, M. (2017). «Hacia una educación universitaria inclusiva: realidad y retos». Revista digital de investigación en docencia universitaria, 11 (1), 4-19.

Alcalá del Olmo, M. J. (2019). «Identidad profesional y formación del docente universitario: retos e incertidumbres.» Márgenes, Revista de Educación de la Universidad de Málaga, 0 (0), 18-37. Disponible en: <http://dx.doi. org/10.24310/mgnmar.v0i0.6201>

Alonso-Sáez, I. y Arandia-Loroño, M. (2017). «15 años desde la Declaración de Bolonia. Desarrollo, situación actual y retos del Espacio Europeo de Educación Superior,» Revista Iberoamericana de Educación Superior, 8 (23), 199-213.

Aparicio, E., Castejón, J. L., Sentana, I., González, A. M., Gras, E., Torregrosa, R. R. y Rico, J. V. (2011). RUA. Redes de investigación docente universitaria: innovaciones metodológicas.

Avolio, B. J., Bass, B. M., Walumbwa, F. y Zhu, W. (2004). Multifactor Leadership Questionnaire: Manual and Sampler Set. Mind Garden, Inc.

Bas, G., Kubiatko, M. y Sunbul, A. M. (2016). «Teachers' perceptions towards ICTs in teaching-learning process: Scale validity and reliability study». Computers in Human Behavior, 61, 176-185. Disponible en: <https://doi. org/10.1016/j.chb.2016.03.022>

BeErkens, E. (2008). «The emergence and institutionalization of the European Higher Education and Research Area». European Journal of Education, 43 (4), 407-425.

Bonilla Barragán, M. D. L., Badillo Gaona, M.y Genis Pérez, E. (2019). «Gestión de la innovación y conocimiento, relación indisoluble para el desarrollo social y bien común en organizaciones educativas». Repositorio de la Red Internacional de Investigadores en Competitividad, 13, 1790-1808. 
Brunet Icart, I. y Vidal Suñé, A. (2004). Empresa y recursos organizativos. Madrid: Pirámide.

Contreras, J. (2018). La autonomía del profesorado. Madrid: Morata.

Englund, C., Olofsson, A. D. y Price, L. (2017). «Teaching with technology in higher education: understanding conceptual change and development in practice». Higher Education Research E Development, 36 (1), 73-87. Disponible en: <https://doi.org/10.1080/07294360.2016.1171300>

Ertmer, P. A., Ottenbreit-Leftwich, A. T., Sadik, O., Sendurur, E. y Sendurur, P. (2012). «Teacher beliefs and technology integration practices: A critical relationship». Computers $E$ Education, 59 (2), 423-435. Disponible en: <https://doi.org/10.1016/j.compedu.2012.02.001>

Esquivel Valverde, Á. F., León Robaina, R. y Castellanos Pallerols, G. M. (2017). «Mejora continua de los procesos de gestión del conocimiento en instituciones de educación superior ecuatorianas». Retos de la Dirección, 11 (2), 56-72.

EURYDICE (2020). The European Higher Education Area in 2020: Bologna Process Implementation Report. European Union.

FAlco, M. (2017). «Reconsiderando las prácticas educativas: TICs en el proceso de enseñanza-aprendizaje». Tendencias pedagógicas, 29, 59-76.

Feeney, S. y Hogan, J. (2017). «A path dependence approach to understanding educational policy harmonisation: the qualifications framework in the European Higher Education Area». Higher Education Policy, 30 (3), 279-298. Disponible en: https://doi.org/10.1057/s41307-016-0019-3

Fernández, J. T. y Pérez, K. V. P. (2018)+ «Nuevos escenarios y competencias digitales docentes: hacia la profesionalización docente con TIC». Profesorado, revista de currículum y formación del profesorado, 22 (1), 25-51.

Fernández-Batanero, J. M. y Ruiz, M. J. C. (2015). "Teacher attitudes towards ICT in the context of inclusive education. Third International Conference on Technological». Ecosystems for Enhancing Multiculturality, 111-115. Disponible en: <https://doi.org/10.1145/2808580.280859>8

Fernández-Ferrer, M. y Miravalles, A. F. (2018). «Evaluación del desarrollo competencial en la educación superior. La perspectiva del profesorado universitario». Educar, 54 (2), 391-410.

García-Cabrero, B., Luna-Serrano, E., Ponce-Ceballos, S., CisnerosCohenour, E., Cordero-Arroyo, G., Espinoza-Díaz, Y. y GarcíaVIGIL, M. H. (2018). «Las competencias docentes en entornos virtuales: Un modelo para su evaluación». Revista Iberoamericana de Educación a Distancia, $21(1), 343-365$. 
García Olmedo, B. (2020). En torno a la universidad española: De los orígenes a la Transición. Vision Libros.

García Peñalvo, F. J. y Rodríguez Conde, M. J. (2020). «Panorámica del eLearning en el sistema universitario español». En G. Ruipérez y J. C. GarCía-Cabrero (eds.). Libro blanco del e-Learning (pp.77-86). Madrid: Bubok.

González, J., Wagenaar, R., Zoller, C., Gehmlich, V., Sticchi-Damiani, M., Isaacs, A. K., Preira, E., Adam, S., Hegarty, A., Doná Della Rose, L. y Smith, A. (2003). Tuning Educational Structures in Europe. Final Report. Phase One. IPARS.

Granda Asencio, L. Y., Espinoza Freire, E. E. y Mayon Espinoza, S. E. (2019). «Las TICs como herramientas didácticas del proceso de enseñanzaaprendizaje». Conrado, 15 (66), 104-110.

Hernández-Ramos, J. P., Martínez-Abad, F., Peñalvo, F. J. G., García, M. E. H. y Rodríguez-Conde, M. J. (2012). «Teacher attitude scale regarding the use of ICT. Reliability and validity study». 2012 International Symposium on Computers in Education (Siie), 6.

Hernández-Sánchez, B. y Sánchez-García, J. C. (eds.) (2018). Educación, desarrollo e innovación social. Andavira.

Herzberg, F. (2017). Motivation to work. Routledge.

Hofstede, G. (1998). «Attitudes, values and organizational culture: Disentangling the concepts». Organization studies, 19 (3), 477-493.

Imbernón, F. (2017). Ser docente en una sociedad compleja: la difícil tarea de enseñar (vol. 50). Graó.

Ion, G., Cano, E*y Cabrera, N. (2016). «Competency Assessment Tool (CAT). The evaluation of an innovative competency-based assessment experience in higher education». Technology Pedagogy and Education, 25 (5), 631-648. Disponible en: <https://doi.org/10.1080/1475939x.2015.1134635>

Jiménez-Hernández, D., González-Ortiz, J. J. y Tornel-Abellán, M. (2018). «Formación del profesorado universitario en metodologías y su incidencia en el aula». Estudios pedagógicos (Valdivia), 44 (3), 157-172.

KaIser, H. (1960). «The application of electronic computers to factor analysis». Educational and Psychological Measurement, 20, 141-151.

Kaiser, H. (1970). «A second generation little jiffy». Psychometrika, 35, 401415.

Koloi-Keaikitse, S. (2017). "Assessment of teacher perceived skill in classroom assessment practices using IRT Models». Cogent Education, 4, 14. Disponible en: <https://doi.org/10.1080/2331186x.2017.1281202> 
Lemos, M., Calle, G., Roldán, T., Valencia, M., Orejuela, J. J. y RománCalderón, J. P. (2019). Factores psicosociales asociados al estrés en profesores universitarios colombianos. Diversitas: Perspectivas en Psicología, 15 (1), $61-72$.

Lucarelli, E. (2004). «Prácticas innovadoras en la formación del docente universitario». Revista Educaçao, 27 (3), 503-524.

Lucas Marín, A. y García Ruiz, P. (2005). Sociología de las organizaciones. México: McGraw-Hill Interamericana.

Llorent, V. J., Zych, I. y Varo-Millán, J. C. (2020). «Competencias socioemocionales autopercibidas en el profesorado universitario en España». Educación XXI, 23 (1).

LunA-Nemecio, J. (2019). «La importancia de la educación para lograr el Desarrollo Social Sostenible. [The Importance of Education to achieve Sustainable Social Development]». Ecocience International Journal, 1 (1), 6-11.

Martínez Lirola, M., Llorens Simón, E. M., Li, H., Rodríguez-Lifante, A., Iniesta, J., Ramos García, A. y Ferreira, A. C. (2020). Hacia una educación universitaria global: diseño de actividades para introducir la Educación para la Ciudadanía Global en la enseñanza universitaria, en Rosabel Roig Vila, Jordi M. Antolí Martínez, Rocío Díez Ros, Neus Pellín Buades (coords.), Memòries del Programa de Xarxes-I3CE de qualitat, innovació $i$ investigació en docència universitària: Convocatòria 2019-20, p. 13-25.

Mishra, P. y Koehler, M. J. (2006). «Technological pedagogical content knowledge: A framework for teacher knowledge». Teachers College Record, 108 (6), 1017-1054. Disponible en: <https://doi.org/10.1111 /j.1467-9620.2006.00684.x>

Moskal, A. C. M., Stein, S. J.y Golding, C. (2016). «Can you increase teacher engagement with evaluation simply by improving the evaluation system?» Assessment E Evaluation in Higher Education, 41 (2), 286-300. Disponible en: <https://doi.org/10.1080/02602938.2015.1007838>

Muñoz-Repiso, A. G. V. y Tejedor, F. J. T. (2012). «The incorporation of ICT in higher education. The contribution of ROC curves in the graphic visualization of differences in the analysis of the variables». British Journal of Educational Technology, 43 (6), 901-919. Disponible en: <https://doi.org/10 $.1111 / \mathrm{j} .1467-8535.2011 .01270+x>$

Muñoz, F. I., Garcia, P. S. y Valenzuela, C. G. (2011). «Teaching skills in virtual and blended learning environments». Comunicar, (36), 107-114. Disponible en: https://doi.org/10.3916/c36-2011-03-01 
Neave, G. (2003). «The Bologna Declaration: Some of the historic dilemmas posed by the reconstruction of the community in Europe's systems of higher education». Educational Policy, 17 (1), 141-164. Disponible en: <https://doi. org/10.1177/0895904802239290>

ONU (2015). Agenda 2030 de Desarrollo Sostenible.

Oros, L. B., Vargas Rubilar, N. y Chemisquy, S. (2020). «Estresores docentes en tiempos de pandemia: un instrumento para su exploración». Revista Interamericana de Psicología, 54 (3).

Pacheco, L. S., Ortega, W. F. A., Chong, E. D. J. D. y Quiñónez, V. M. R. (2017). «Las Tics en los procesos de enseñanza y aprendizaje en la educación universitaria». Dominio de las Ciencias, 3 (2), 721-749.

Peralvo, C. del R., Arias, P. A. y Merino, M. M. (2018). «Retos de la docencia universitaria en el siglo xxI». Revista Órbita Pedagógica, 5 (1), 09-27.

Perrow, Ch. (1990). Sociología de las Organizaciones. Madrid: McGraw-Hill.

Peralta Miranda, P., Stefanel Santiago, I. C., Cervantes Atia, V. y Salgado Herrera, R. P. (2018). «Calidad de servicio en una institución de educación superior en la ciudad de Barranquilla». Ciencias Administrativas, (11), 017-017.

Prosser, M., Martin, E., Trigwell, K., Ramsden, P. y Lueckenhausen, G. (2005). "Academics' experiences of understanding of their subject matter and the relationship of this to their experiences of teaching and learning». Instructional Science, 33 (2), 137-157. Disponible en: <https://doi. org/10.1007/s11251-004-7687-x>

Quiroz, D. L. Z. y Quiroz, M. S. Z. (2019). «Las tecnologías de la información y las comunicaciones (TICs) en la Educación Superior: Consideraciones teóricas». REFCalE: Revista Electrónica Formación y Calidad Educativa, 7 (1), 213-228.

Regueira, U. y Gewerc, A. (2020). «¿Qué ha cambiado desde la implementación del EEES? Estudio de la percepción del profesorado universitario de Galicia sobre la enseñanza», en Rosabel Roig Vila (coord.), La docencia en la Enseñanza Superior: Nuevas aportaciones desde la investigación e innovación educativas, p. 385-395.

Rodríguez-Gómez, R. (2019). «Autonomía universitaria y rendición de cuentas». Revista mexicana de investigación educativa, 24 (82), 879-895.

Romero-Martín, M. R., Castejón-Oliva, F. J., López-Pastor, V. M. y Fraile-Aranda, A. (2017). «Formative assessment, communication skills and ICT in initial teacher training». Comunicar, (52), 73-82. Disponible en: <https://doi.org/10.3916/c52-2017-07> 
Salgueiro, M. A., Sabelli, M. J., Manzi, M. L., Irigoyen, R. A., Exeni, C. H., Vidal, M. B. B. y Bono, L. C. (2021). Planificar, enseñar, aprender y evaluar en educación superior: Nuevos entornos integrados de aprendizajes. De la presencialidad a la virtualidad (vol. 26). Noveduc.

Sanagustín-Fons, M. V., Puyal, E., Moseñe, J. A. y Tricas, J. M. (2007). El Espacio Europeo de Educación Superior, nuevos planes de estudio y presencia de la sociología de las organizaciones en los mismos. Zaragoza.

Sanagustín-Fons, M., Cruz Bello, F. y Paricio Royo, J. (2008). Investigación Educativa e Innovación Docente en el proceso de Convergencia Europea (No. BOOK-2020-072). Prensas Universitarias de Zaragoza.

Sanagustín Fons, M. V. (2012). Valores y ética empresarial: Un enfoque sociológico. Madrid: Trotta.

Sanagustín-Fons, M. V. y Brunet Icart, I. (2017). «Modelo de innovación socio-institucional en la administración pública». Revista internacional de Organizaciones, (19), 93-116.

Sánchez, O. M., Amar, R. M. y Triadú, J. X. (2018)+ «Habilidades blandas: necesarias para la formación integral del estudiante universitario». Revista Cientifica Ecociencia, 5, 1-18.

Saroyan, A. y Trigwell, K. (2015). «Higher education teachers' professional learning: Process and outcome». Studies in Educational Evaluation, 46, 92 101. Disponible en: <https://doi.org/10.1016/j.stueduc.2015.03.008>

Schein, E. H. (2010). Organizational culture and leadership (vol. 2). John Wiley $\&$ Sons.

Scherer, R., Siddie, F. y Teo, T. (2015). «Becoming more specific* Measuring and modeling teachers' perceived usefulness of ICT in the context of teaching and learning». Computers $\&$ Education, 88 (Supplement C), 202-214. Disponible en: <https //doi.org/https;//doi.org/10.1016/j. compedu.2015.05.005>

Sisto, V. (2020). «Desbordadas/os: Rendición de Cuentas e Intensificación del Trabajo en la Universidad Neoliberal. El Caso de Chile». Education Policy Analysis Archives, 28.

Stes, A., Min-Leliveld, M., Gijbels, D. y Van Petegem, P. (2010). «The impact of instructional development in higher education: The state-of-theart of the research». Educational Research Review, 5 (1), 25-49. Disponible en: https://doi.org/https://doi.org/10.1016/j.edurev+2009.07.001

Surdez Pérez, E. G., Magaña Medina, D. E. y Sandoval Caraveo, M. D. C. (2017). «Evidencias de ambigüedad de rol en profesores universitarios». Revista electrónica de investigación educativa, 19 (1), 73-83. 
Tacca Huamán, D. R., Tacca Huamán, A. L. y Cuarez Cordero, R. (2020). «Inteligencia emocional del docente y satisfacción académica del estudiante universitario». Revista Digital de Investigación en Docencia Universitaria, 14 (1).

Tejedor, F. J., García-Valcárcel, A. y Prada, S. (2009). "A scale for the measurement of University teachers' attitudes towards the integration of ICT». Comunicar, (33), 115-124. Disponible en* <https://doi.org/10.3916/ c33-2009-03-002>

Tigelaar, D. E. H., Dolmans, D., Wolfhagen, I. y Van der Vleuten, C. P. M. (2004). «The development and validation of a framework for teaching competencies in higher education». Higher Education, 48 (2), 253-268. Disponible en: <https://doi.org/10.1023/B:HIGH.0000034318.74275.e4>. Assessment E Evaluation in Higher Education, 37 (4), 499-511. Disponible en: <https://doi.org/10.1080/02602938.2010.54792>9

VAN den Bos, P. y Brouwer, J. (2014), «Learning to teach in higher education: how to link theory and practice.» Teaching in Higher Education, 19 (7), 772 786. Disponible en: <https;//doi.org/10.1080/13562517.2014.901952>

Van der Lans, R. M., VAn de Grift, W. y Van Veen, K. (2015). «Developing a teacher evaluation instrument to provide formative feedback using student ratings of teaching acts». Educational Measurement-Issues and Practice, 34 (3), 18-27. Disponible en: <https://doi.org/10.1111/emip.12078>

VAN der Wende, M. C. (2000). «The Bologna Declaration: enhancing the transparency and competitiveness of European higher education». Higher Education in Europe, 25, 305-310.

Vergara, M. V. y Núñez, L. A. (2021). Los Objetivos de Desarrollo Sostenible: boja de ruta en la educación del siglo XXI: Innovación docente en la formación de profesionales. Octaedro.

Villarroel, V. A. y Bruna, D. V. (2017). «Competencias pedagógicas que caracterizan a un docente universitario de excelencia: un estudio de caso que incorpora la perspectiva de docentes y estudiantes». Formación universitaria, 10 (4), 75-96.

Visser-Wijnveen, G. J., Stes, A. Y Van Petegem, P. (2011). «Development and validation of a questionnaire measuring teachers' motivations for teaching in higher education». Pedagogische Studien, 88 (6), 383-399.

Walker, V. S. (2015). «Aportes teóricos para pensar el trabajo docente en la universidad». Actualidades Investigativas en Educación, 15 (1), 564-584.

Walker, V. S. (2020). «Tendencias en el campo de la educación superior y su incidencia en el Trabajo Docente Universitario». Revista de la educación superior, 49 (193), 107-127. 
Wilkerson, L.e Irby, D.M.(1998). «Strategies for improving teaching practices: A comprehensive approach to faculty development». Academic Medicine, 73 (4), 387-396. Disponible en: <https://doi.org/10.1097/00001888199804000-00011>

Wilson, S. M. y Berne, J. (1999). "Teacher learning and the acquisition of professional knowledge: An examination of research on contemporary professional development». Review of Research in Education, 24, 173-209.

Zabalza, M. Á. y Beraza, M. Á. Z. (2003). Competencias docentes del profesorado universitario: calidad y desarrollo profesional (vol. 4). Narcea. 